\title{
Combined effect of initial biomass density and nitrogen concentration on growth and astaxanthin production of Haematococcus pluvialis (Chlorophyta) in outdoor cultivation
}

\author{
Junfeng Wang ${ }^{1,2, a}$, Milton R. Sommerfeld ${ }^{1}$, Congming $\mathrm{Lu}^{2}$ and Qiang $\mathrm{Hu}^{1, *}$ \\ ${ }^{1}$ Laboratory for Algae Research and Biotechnology, College of Technology and Innovation, Arizona State University \\ Polytechnic Campus, Mesa, AZ 85212, USA \\ ${ }^{2}$ Photosynthesis Research Center, Key Laboratory of Photobiology, Institute of Botany, Chinese Academy of Sciences, \\ Beijing 100093, China
}

\begin{abstract}
Nitrogen availability and cell density each affects growth and cellular astaxanthin content of Haematococcus pluvialis, but possible combined effects of these two factors on the content and productivity of astaxanthin, especially under outdoor culture conditions, is less understood. In this study, the effects of the initial biomass densities IBDs of 0.1, 0.5, 0.8, $1.5,2.7,3.5$, and $5.0 \mathrm{~g} \mathrm{~L}^{-1} \mathrm{DW}$ and initial nitrogen concentrations of $0,4.4,8.8$, and $17.6 \mathrm{mM}$ nitrate on growth and cellular astaxanthin content of $H$. pluvialis Flotow K-0084 were investigated in outdoor glass column photobioreactors in a batch culture mode. A low IBD of $0.1 \mathrm{~g} \mathrm{~L}^{-1} \mathrm{DW}$ led to photo-bleaching of the culture within 1-2 days. When the IBD was $0.5 \mathrm{~g} \mathrm{~L}^{-1}$ and above, the rate at which the increase in biomass density and the astaxanthin content on a per cell basis was higher at lower IBD. When the IBD was optimal (i.e., $0.8 \mathrm{~g} \mathrm{~L}^{-1}$ ), the maximum astaxanthin content of $3.8 \%$ of DW was obtained in the absence of nitrogen, whereas the maximum astaxanthin productivity of $16.0 \mathrm{mg} \mathrm{L}^{-1} \mathrm{~d}^{-1}$ was obtained in the same IBD culture containing $4.4 \mathrm{mM}$ nitrogen. The strategies for achieving maximum Haematococcus biomass productivity and for maximum cellular astaxanthin content are discussed.
\end{abstract}

Key Words: astaxanthin; cell density; Haematococcus pluvialis; nitrogen; photobioreactor; outdoor culture

\section{INTRODUCTION}

The green microalga, Haematococcus pluvialis Flotow (Chlorophyceae), is considered to be the richest natural source of astaxanthin for pharmaceutical, nutraceutical, and cosmetics applications due to its high antioxidant activities and for the aquaculture and poultry industries due to its impact on animal pigmentation (Miki 1991, Nishino 1998, Kobayashi 2000, Guerin et al. 2003, Karppi et al. 2007). Mass cultivation of H. pluvialis for commercial purposes has been exploited in both open ponds (Johnson and An 1991, Zhang et al. 2009) and closed pho- tobioreactors of various designs since the 1990's (Lorenz and Cysewski 2000, Han et al. 2013). Light and nitrogen are the two most effective factors that influence growth and astaxanthin synthesis in this organism. Because the light and nitrogen requirements for growth are different from that for astaxanthin synthesis, a two-step cultivation strategy is commonly adopted in which the first step is to promote algal growth under favorable culture conditions (e.g., low light and nitrogen-replete). When a high, yet optimized cell density is reached, the culture enters (c) This is an Open Access article distributed under the terms of the Creative Commons Attribution Non-Commercial License (http://creativecommons.org/licenses/by-nc/3.0/) which permits unrestricted non-commercial use, distribution, and reproduction in any medium, provided the original work is properly cited.

Copyright $@$ The Korean Society of Phycology
Received April 15, 2013, Accepted May 10, 2013

*Corresponding Author

E-mail: huqiang@asu.edu

Tel: +1-480-727-1484, Fax: +1-480-727-1236

${ }^{a}$ Present address: Qingdao Institute of Bioenergy and Bioprocess Technology, Chinese Academy of Sciences, Qingdao, Shandong, 266101, China 
into the second step where algal cells are subjected to stress conditions (e.g., high light and nitrogen deplete) for astaxanthin production (Borowitzka et al. 1991, Boussiba and Vonshak 1991, Lorenz and Cysewski 2000, Fábregas et al. 2001, Torzillo et al. 2003, Aflalo et al. 2007). As Haematococcus cells are green in the first step and red in the second one, the first step is also called the 'green stage' and the second step is called the 'red stage'.

In a previous study, we demonstrated that a record high astaxanthin productivity of $17.1 \mathrm{mg} \mathrm{L}^{-1} \mathrm{~d}^{-1}$ can be obtained from $H$. pluvialis culture in an outdoor glass column photobioreactor in which high nitrogen $(17.6 \mathrm{mM}$ nitrate-N) was supplied and the initial biomass density (IBD) was optimized (Wang et al. 2013). The work suggested that nitrogen, although is a critical factor, does not work alone, but interacts with other factors, such as light intensity, to affect overall astaxanthin production. Likewise, the effect of light intensity on astaxanthin production can be largely altered by algal density in the culture, as the latter affects the light availability to individual cells under a given incident light intensity (Hu and Richmond 1996, Hu et al. 1998). In order to maximize astaxanthin production in $H$. pluvialis culture, an optimal nitrogen concentration (rather than the lower the better) and light intensity (rather than the higher the better) must be determined.

In this study, the combined effect of nitrogen concentration and algal biomass density (which affects light intensity at the single cell level) on astaxanthin production potential was investigated in $H$. pluvialis grown in an outdoor glass column photobioreactor. First, the effect of IBD on astaxanthin production under nitrogen deplete condition was determined. The optimal IBD identified served as a baseline upon which the effect of different initial nitrogen concentrations on astaxanthin production was then assessed. The results indicated that it was the optimized IBD under nitrogen limited (i.e., $4.4 \mathrm{mM}$ nitrate), rather than nitrogen-deplete or nitrogen-replete conditions that resulted in the highest astaxanthin productivity.

\section{MATERIALS AND METHODS}

Haematococcus pluvialis Flotow K-0084 was obtained from the Scandinavian Culture Collection of Algae and Protozoa at the University of Copenhagen, Denmark and maintained in BG11 culture medium (Stanier et al. 1971). Cultures were conducted in glass column photobioreactors each measuring $0.7 \mathrm{~m}$ height and $4.2 \mathrm{~cm}$ inner di- ameter with a working volume of $0.6 \mathrm{~L}$. The culture was continuously bubbled with compressed air containing $1.5 \%(\mathrm{v} / \mathrm{v})$ of $\mathrm{CO}_{2}$ to provide sufficient amounts of carbon for photosynthesis while maintaining $\mathrm{pH}$ at 7-8. Green stage cultivation was conducted under laboratory conditions $\left(20^{\circ} \mathrm{C}\right.$, continuous illumination of $\left.30 \mu \mathrm{mol} \mathrm{m}^{-2} \mathrm{~s}^{-1}\right)$. Cells in the late log phase (10-12 days old) was used as "seed" for outdoor experiments which were carried out at Arizona State University Polytechnic campus in Mesa, Arizona, USA ( $\left.33^{\circ} 24^{\prime} \mathrm{N}, 111^{\circ} 49^{\prime} \mathrm{W}\right)$ from March 7 through May 18, 2009. The highest air temperatures during day time were $25.5^{\circ} \mathrm{C}$ in March and $32.2^{\circ} \mathrm{C}$ in May, while the lowest air temperatures during night time were $7.2^{\circ} \mathrm{C}$ in March and $14.4^{\circ} \mathrm{C}$ in May. The monthly average precipitation was less than $10 \mathrm{~mm}$ during the time period when the experiments were conducted. The light intensity measured on the surface of the photobioreactors during the daylight period followed a more or less symmetric double peak curve fashion with the first peak value of 1,900-2,000 $\mathrm{mmol} \mathrm{m}^{-2} \mathrm{~s}^{-1}$ measured at 9:00-10:00 and the second peak value of 1,400-1,700 $\mu \mathrm{mol} \mathrm{m}^{-2} \mathrm{~s}^{-1}$ at 14:0017:00. Sixteen columns were arranged side by side with an east-west orientation. Culture temperature was maintained between $20-25^{\circ} \mathrm{C}$ during the daylight period by an evaporative cooling system.

For the IBD experiment, the culture medium was free of nitrogen, and the IBDs were set as 0.1, 0.5, 0.8, 1.5, 2.7, 3.5, and $5.0 \mathrm{~g} \mathrm{~L}^{-1} \mathrm{DW}$. For the nitrogen concentration experiment, the BG-11 medium containing 0, 0.375, 0.75, and $1.5 \mathrm{~g} \mathrm{~L}^{-1}$ sodium nitrate were prepared, which corresponded to the $0,4.4,8.8,17.6 \mathrm{mM}$ nitrate, respectively. More than $90 \%$ of the cells for inoculation were in a palmella stage (non-flagellated green vegetative cells) and less than $10 \%$ were green swimming cells with two flagella. The average chlorophyll content of inoculum cells was $3.0 \pm 0.8 \% \mathrm{DW}$. The maximum photochemical efficiency of photosystem II $\left(F_{\mathrm{v}} / F_{\mathrm{m}}\right)$, as measured by Dual-Pam-100 (Walz, Effeltrich, Germany) (Stirbet and Govindjee 2011, Wang et al. 2013), was ca. 0.7, indicative of a healthy status of the cells used for outdoor experiments.

Algal biomass concentration was measured by a gravimetric method. Briefly, an aliquot of culture sample $(v$, 5-20 mL) was filtered through a pre-weighed glass fiber filter (GF/F, pore size $0.4 \mu \mathrm{m}$; Whatman, Kent, UK), which was then rinsed with $50 \mathrm{~mL}$ de-ionized water to remove any remaining salts on the algae and filter. The filter containing algal cells was dried in an oven at $105^{\circ} \mathrm{C}$ overnight to a constant weight. The biomass concentration of the sample DW ( $\left.\mathrm{g} \mathrm{L}^{-1}\right)$ was calculated as: 


$$
\mathrm{DW}=\left(\mathrm{W}_{1}-\mathrm{W}_{0}\right) \times 1,000 / v
$$

, where $W_{1}$ and $W_{0}$ represent the weights of the filter paper with and without the algae (g), respectively. Specific growth rate $\left(\mu, d^{-1}\right)$ was calculated as:

$$
\mu=\left[\ln \left(\mathrm{DW}_{\mathrm{t}}\right)-\ln \left(\mathrm{DW}_{0}\right)\right] / \mathrm{t}
$$

, where $t$ represents the cultivation time at the red stage. The biomass productivity $\left(\mathrm{g} \mathrm{L}^{-1} \mathrm{~d}^{-1}\right)$ was calculated as:

$$
\text { Biomass productivity }=\left(\mathrm{DW}_{\mathrm{t}}-\mathrm{DW}_{0}\right) / \mathrm{t}
$$

For red stage biomass productivity, $\mathrm{DW}_{\mathrm{t}}$ and $\mathrm{DW}_{0}$ represent biomass densities at day $\mathrm{t}$ and day 0 of red stage, respectively, and $t$ represents the cultivation time of the red stage. For overall biomass productivity, $\mathrm{DW}_{\mathrm{t}}$ and $\mathrm{DW}_{0}$ represent biomass densities at day $t$ of the red stage and biomass at day 0 of the green stage, respectively and $t$ represents the entire cultivation time (green stage plus red stage). An average biomass productivity of the green stage culture of $H$. pluvialis was $0.1 \mathrm{~g} \mathrm{~L}^{-1} \mathrm{~d}^{-1}$.

The cellular astaxanthin content was measured by reverse-phase high performance liquid chromatography according to Wang et al. (2013). Chlorophyll concentration of algal samples was calculated according to Lichtenthaler and Wellburn (1983). Optical densities at 647 and $663 \mathrm{~nm}$ were measured using a spectrophotometer (DU650; Beckman and Coulter, Fullerton, CA, USA).

The nitrogen concentration of the culture (in the form of sodium nitrate) was measured by Quikchem8500 (Lachat, Milwaukee, WI, USA) according to the manufacturer's instructions. Aliquots of $10 \mathrm{~mL}$ culture were centrifuged at 10,000 $\times \mathrm{g}$ for $10 \mathrm{~min}$, and the supernatants saved for nitrate analysis.

\section{RESULTS AND DISCUSSION}

In our previous study we demonstrated that under nitrogen-replete conditions the $H$. pluvialis cultures varying in IBD (i.e., 0.5, 0.8, 1.5, 2.7, 3.5, and $5.0 \mathrm{~g} \mathrm{~L}^{-1} \mathrm{DW}$ ) exhibited a similar growth pattern and reached more or less the same final biomass density $\left(4.1 \pm 0.4 \mathrm{~g} \mathrm{~L}^{-1} \mathrm{DW}\right)$ by the end of a 10 day red stage cultivation. As increasing IBD from 0.5 to $5.0 \mathrm{~g} \mathrm{~L}^{-1}$, the rate of increase in the cellular astaxanthin content decreased. As a result, the highest astaxanthin content of $2.5 \%$ of dry weight occurred in the $0.5 \mathrm{~g} \mathrm{~L}^{-1}$ IBD cultures, whereas the lowest astaxanthin content of $0.5 \%$ was measured in the $5.0 \mathrm{~g} \mathrm{~L}^{-1} \mathrm{IBD}$ cul- tures. Little biomass increase and a low astaxanthin content of ca. $0.5 \%$ were obtained in the $0.1 \mathrm{~g} \mathrm{~L}^{-1} \mathrm{IBD}$ cultures (Wang et al. 2013).

In this study, we took an additional step to determine whether the relationship between IBD and growth and astaxanthin content was influenced by nitrogen depletion. We utilized the same experimental setting used in the previous study except that at the red stage no nitrate was provided to the cultures. The results showed that the maximum specific growth rate of $H$. pluvialis cultures varying in IBDs from $0.5,0.8,1.5,2.7,3.5$ to $5.0 \mathrm{~g} \mathrm{~L}^{-1}$ occurred on day 1 of cultivation, and yet the lower the IBD, the higher the maximum specific growth rate obtained. The maximum specific growth rate of $0.6 \mathrm{~g} \mathrm{~d}^{-1}$ obtained in the 0.5 and $0.8 \mathrm{~g} \mathrm{~L}^{-1}$ IBD cultures (Fig. 1A) was comparable to that achieved under nitrogen-replete conditions (Wang et al. 2013). As the cultivation continued, the specific growth rates decreased, albeit at different rates. The lower the IBD the more rapid reduction in specific growth rate.

The biomass densities of the different IBD cultures increased gradually during the red stage with more biomass accumulation in the cultures of higher IBDs (Fig. 1B). However, when the biomass increases in the individual cultures were calculated on a per IBD basis, a reverse relationship between IBD and biomass productivity was evident. The higher IBDs resulted in lower net biomass yields (Fig. 1C). When the IBD was $0.1 \mathrm{~g} \mathrm{~L}^{-1}$, the cells died and the cultures bleached in one or two days due to high light-induced photooxidative cell death (Han et al. 2012).

When the IBD was $0.5 \mathrm{~g} \mathrm{~L}^{-1}$ or higher, the cellular astaxanthin content increased gradually and reached the highest level on day 10, and thereafter it remained the same or declined. A clear trend was that the lower the IBD the higher the rate of astaxanthin accumulation and thus the higher cellular astaxanthin content. As a result, the highest astaxanthin content of $3.3 \%$ dry weight was obtained in the $0.5 \mathrm{~g} \mathrm{~L}^{-1}$ IBD cultures (Fig. 1D).

Based on the above and our previous experiments (Wang et al. 2013), a general trend was observed that for a given IBD, a nitrogen-deplete culture reduces the final biomass density but increases the maximum cellular astaxanthin content. Regardless of the nitrogen status, the optimal IBD of 0.5-0.8 $\mathrm{g} \mathrm{L}^{-1} \mathrm{DW}$ was higher than the $0.29 \mathrm{~g} \mathrm{~L}^{-1}$ applied to an outdoor horizontal tubular photobioreactor ( $4.85 \mathrm{~cm}$ inner diameter, $50 \mathrm{~L}$ culture volume) (Torzillo et al. 2003) and $0.4 \mathrm{~g} \mathrm{~L}^{-1}$ in an outdoor horizontal airlift tubular $(30 \mathrm{~mm}$ inner diameter, $55 \mathrm{~L}$ culture volume) and an outdoor vertical bubble column photobioreactor (20 cm inner diameter, 55 L culture volume) 

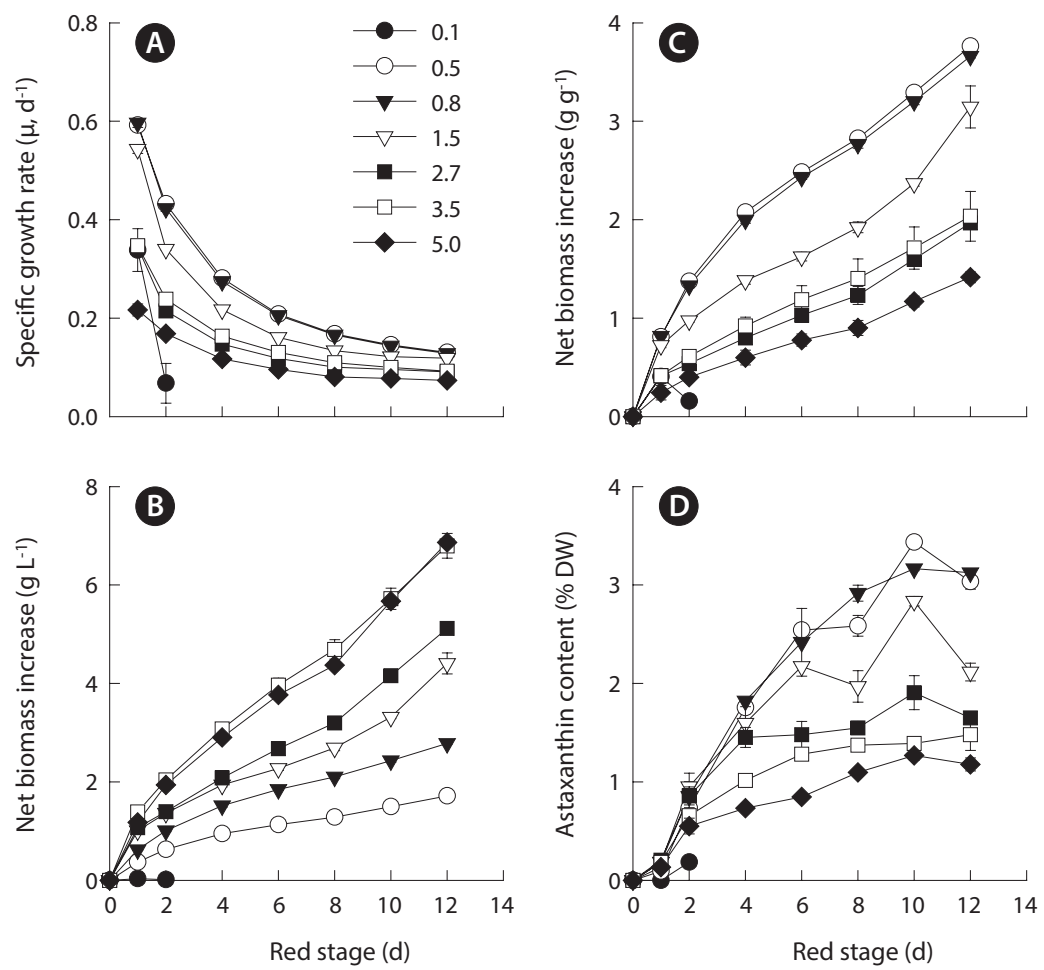

Fig. 1. Effects of initial biomass densities (IBDs) on the growth and astaxanthin content of Haematococcus pluvialis in nitrogen-deplete culture medium under outdoor conditions. (A) The specific growth rate $\left(\mu \mathrm{d}^{-1}\right)$ as a function of IBD and time at the red stage. (B) Net volumetric biomass increase $\left(\mathrm{g} \mathrm{L}^{-1}\right)$ as a function of IBD and time at the red stage. (C) Net biomass increase $\left(\mathrm{g} \mathrm{g}^{-1}\right)$ as a function of time on a per IBD basis. (D) Cellular astaxanthin content (\% DW) as a function of IBD and time at the red stage. Data were means \pm standard deviations of six measurements from three individual columns (two measurements per column). The values in the legend represent different IBD treatments $\left(\mathrm{g} \mathrm{L}^{-1}\right)$.

(García-Malea López et al. 2006), but lower than that (1.3 $\left.\mathrm{g} \mathrm{L}^{-1}\right)$ started in an outdoor horizontal tubular photobioreactor (30 mm inner diameter, 2,000 L culture volume) (Aflalo et al. 2007).

The maximum volumetric biomass productivity in the red stage occurred on day 1 of cultivation. As the IBD increased from 0.5 to $3.5 \mathrm{~g} \mathrm{~L}^{-1}$, the maximum volumetric biomass productivity increased from $0.37 \pm 0.01$ to $1.39 \pm$ $0.02 \mathrm{~g} \mathrm{~L}^{-1} \mathrm{~d}^{-1}$. Further increasing in IBD resulted in a slightly reduced productivity. As the cultivation proceeded, the volumetric biomass productivities of all the treatments decreased with distinct patterns, where more dramatic decreases in productivity occurred in the cultures of higher IBDs (Fig. 2A).

Because the volumetric astaxanthin productivity is a function of biomass density and the cellular astaxanthin content, the trend for astaxanthin productivity was somewhat different from that of biomass productivity. As shown in Fig. 2B, the maximum volumetric astaxanthin productivity at the red stage increased from $6.14 \pm 0.19$ $\mathrm{mg} \mathrm{L}^{-1} \mathrm{~d}^{-1}$ in the IBD $0.5 \mathrm{~g} \mathrm{~L}^{-1}$ cultures, to $10.51 \pm 1.49 \mathrm{mg}$ $\mathrm{L}^{-1} \mathrm{~d}^{-1}$ in the IBD $0.8 \mathrm{~g} \mathrm{~L}^{-1}$ cultures, to $13.31 \pm 1.41 \mathrm{mg} \mathrm{L}^{-1} \mathrm{~d}^{-1}$ in the IBD $1.5 \mathrm{~g} \mathrm{~L}^{-1}$ cultures, and to $18.71 \pm 2.69 \mathrm{mg} \mathrm{L}^{-1} \mathrm{~d}^{-1}$ in the IBD $5.0 \mathrm{~g} \mathrm{~L}^{-1}$ cultures. The volumetric astaxanthin productivity of $18.71 \pm 2.69 \mathrm{mg} \mathrm{L}^{-1} \mathrm{~d}^{-1}$ was the highest ever achieved at the red stage of outdoor $H$. pluvialis cultures. It is worth noting that the maximum astaxanthin productivity occurred within the first 2 to 4 days when the cellular astaxanthin content was just about half of the maximum attainable. When the cellular astaxanthin content reached the highest level at each IBD on day 10, the volumetric astaxanthin productivity either remained stable at the low IBDs $\left(0.5,0.8\right.$, and $\left.1.5 \mathrm{~g} \mathrm{~L}^{-1} \mathrm{DW}\right)$ or decreased considerably at the high IBDs $\left(2.7,3.5\right.$, and $\left.5.0 \mathrm{~g} \mathrm{~L}^{-1} \mathrm{DW}\right)$.

Since the red stage was part of the Haematococcus cultivation process for astaxanthin production, the time spent at the green stage should be taken into account when determining overall productivity. Under our experimental conditions, the average biomass productivity of $\mathrm{H}$. pluvialis at the green stage was $0.1 \mathrm{~g} \mathrm{~L}^{-1} \mathrm{~d}^{-1}$. Accordingly, the time for preparing the inoculum densities of 0.1, 0.5, 0.8, $1.5,2.7,3.5$, and $5.0 \mathrm{~g} \mathrm{~L}^{-1} \mathrm{DW}$ for outdoor red stage experiments were $1,5,8,15,27,35$, and 50 days, respectively.

During the entire cultivation period (viz. green stage 

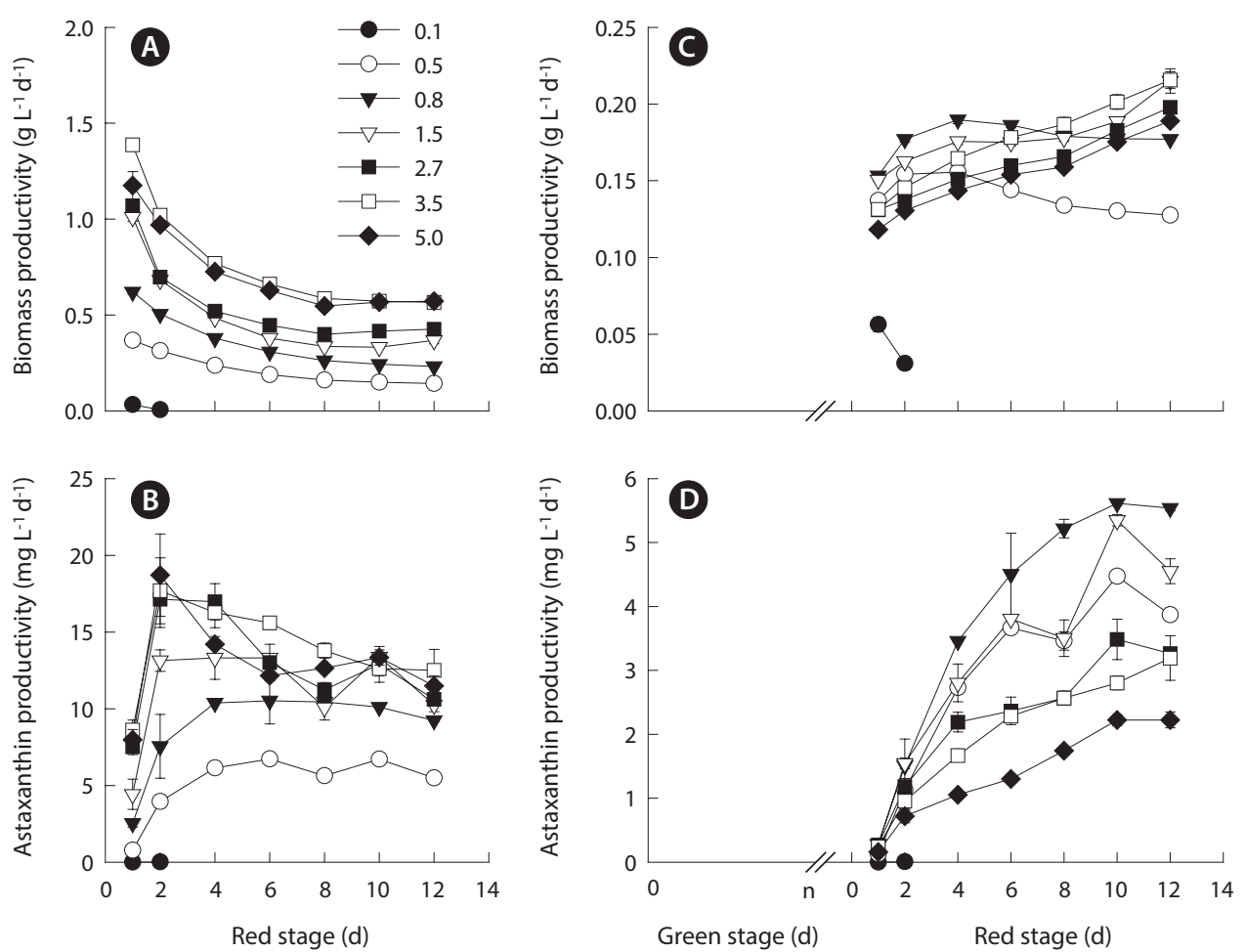

Fig. 2. Effects of different initial biomass densities (IBDs) on the biomass productivity and astaxanthin productivity of Haematococcus pluvialis in nitrogen-deplete culture medium under outdoor conditions. (A \& B) Results on the red stage. (C \& D) Results on the green + red stages. The average biomass productivity of green stage culture of $H$. pluvialis was $0.1 \mathrm{~g} \mathrm{~L}^{-1} \mathrm{~d}^{-1}$. Accordingly, the time $(n)$ for preparing the inoculum densities of $0.1,0.5,0.8,1.5,2.7,3.5$, and $5.0 \mathrm{~g} \mathrm{~L}^{-1} \mathrm{DW}$ were calculated to be $\mathrm{n}=1,5,8,15,27,35$, and 50 days, respectively. Data were means \pm standard deviations of six measurements from three individual columns (two measurements per column). The values in the legend represent different IBD treatments $\left(\mathrm{g} \mathrm{L}^{-1}\right)$.

plus red stage) the volumetric biomass productivity of the different IBD cultures decreased dramatically. The time required for achieving maximum biomass productivity was also changed. When the IBD was low and moderate $\left(0.5,0.8\right.$, and $\left.1.5 \mathrm{~g} \mathrm{~L}^{-1} \mathrm{DW}\right)$, the maximum biomass productivity occurred within the first 2 to 4 days of the red stage. In contrast, the maximum biomass productivity was obtained at the end of the red stage when the IBDs were high (2.7, 3.5, and $5.0 \mathrm{~g} \mathrm{~L}^{-1} \mathrm{DW}$ ) (Fig. 2C).

Likewise, when the green stage was taken into account, the overall volumetric astaxanthin productivities of the different IBD cultures were reduced considerably as compared to that obtained from the red stage only. However, the trend of volumetric astaxanthin productivity as a function of IBD was drastically different from that obtained from the red stage. The maximum astaxanthin productivity of $4.47 \pm 0.03 \mathrm{mg} \mathrm{L}^{-1} \mathrm{~d}^{-1}$ was obtained in the IBD $0.5 \mathrm{~g} \mathrm{~L}^{-1}$ cultures, and it increased to $5.61 \pm 0.03 \mathrm{mg}$ $\mathrm{L}^{-1} \mathrm{~d}^{-1}$ when the IBD was raised to $0.8 \mathrm{~g} \mathrm{~L}^{-1}$, above which the higher the IBD the lower the maximum astaxanthin productivity (Fig. 2D).

Because the nitrogen availability for achieving maxi- mum growth was mutually exclusive from achieving maximum cellular astaxanthin content, we selected $0.8 \mathrm{~g}$ $\mathrm{L}^{-1}$ as the optimal IBD to further optimize the initial nitrogen concentration for maximum astaxanthin production. To this end, the initial nitrogen concentrations of 0 , 4.4, 8.8, and $17.6 \mathrm{mM}$ nitrate were chosen and the results are shown in Fig. 3A. At the red stage the nitrate concentrations in the 4.4, 8.8, and $17.6 \mathrm{mM}$ nitrate cultures decreased at more or less the same rate, indicating that Haematococcus cells exhibited the same cellular nitrate uptake rate when the initial nitrogen concentration in the cultures reached a threshold level (ca. $4 \mathrm{mM}$ nitrate). The depletion of nitrate in the 4.4 and $8.8 \mathrm{mM}$ nitrate cultures occurred on day 2 and 4, respectively. In the $17.6 \mathrm{mM}$ nitrate- $\mathrm{N}$ cultures, the nitrate concentration decreased to $3.2 \mathrm{mM}$ on day 6 and remained constant thereafter (Fig. $3 A)$. The initial nitrate concentration did affect the final biomass density, i.e., the higher the initial nitrogen concentration in the culture, the greater the final biomass density at the end of the 10 day culture period (Fig. 3B). The increases in final biomass density were due to the increases in both cell number and cell size. As shown in 

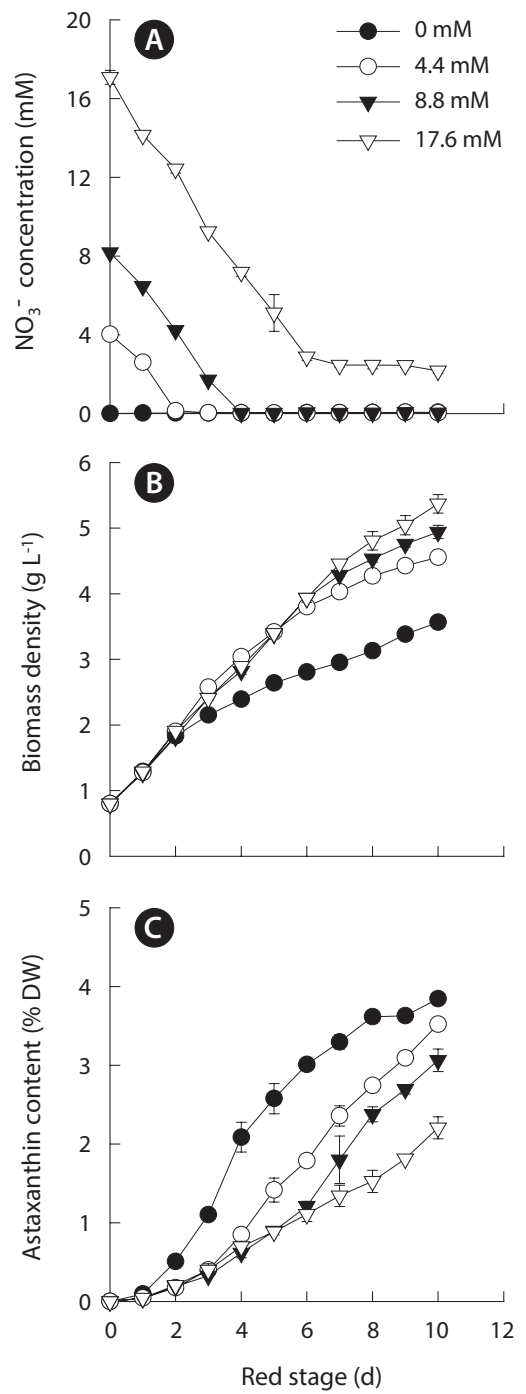

Fig. 3. Effects of nitrate concentrations on nitrate uptake kinetics (A), growth (B), and astaxanthin content (C) of Haematococcus pluvialis under outdoor conditions. Data were means \pm standard deviations of six measurements from three individual columns (two measurements per column). The values in the legend represent different initial nitrogen concentration treatments. The initial biomass density was $0.8 \mathrm{~g} \mathrm{~L}^{-1}$ for this experiment.

Fig. 4, Haematococcus cells underwent both reproduction and cell enlargement throughout the red stage. We further observed that under nitrogen-deplete conditions, the increase in biomass density was mainly contributed by the increase in cell size, and to a lesser extent cell reproduction. Conversely, it was cell reproduction and to a lesser extent cell enlargement that contributed to biomass increase in the nitrogen-replete cultures. At the end of the red stage, the average diameter of red cysts in the nitrogen-depleted and $4.4 \mathrm{mM}$ nitrate cultures was $28 \pm 6$ $\mu \mathrm{m}$, whereas that of red cysts was $18 \pm 6 \mu \mathrm{m}$ in the 8.8 and $17.6 \mathrm{mM}$ nitrate cultures.

A reverse relationship between the initial nitrogen concentration and the maximum cellular astaxanthin content was observed, i.e., the lower the initial nitrogen concentration, the more rapid increase in cellular astaxanthin content and the higher the maximum cellular astaxanthin content. The highest cellular astaxanthin content of $3.84 \pm 0.05 \%$ DW was obtained in the nitrogendepleted cultures, whereas the lowest astaxanthin content of $2.20 \pm 0.14 \%$ DW was measured in the $17.6 \mathrm{mM}$ nitrate cultures (Fig. 3C).

The volumetric biomass productivity as a function of initial nitrogen concentration is shown in Fig. 5A. The fact that the biomass productivity of the nitrate-deplete cultures were identical to that obtained in the nitratelimited or nitrogen-replete cultures for the first two days suggested that the amount of intracellular nitrogen was sufficient to sustain initial vigorous growth. The highest biomass productivity of $0.59 \pm 0.01 \mathrm{~g} \mathrm{~L}^{-1} \mathrm{~d}^{-1}$ was obtained in the $4.4 \mathrm{mM}$ nitrate cultures. The culture with initial nitrate concentration that was lower or higher than this level resulted in reduced biomass productivity. A possible interpretation is that during the transformation of $\mathrm{Hae}$ matococcus cells, particularly in the early process, from green vegetative cells to red cysts, the cells require a certain amount of nitrogen, which may be too low to sustain normal growth and reproduction but high enough to help augment cellular enzymatic defense systems to cope with high light stress. As a result, the cells can achieve a certain degree of growth / biomass production while synthesizing a large amount of astaxanthin as part of a molecular defense mechanism to protect the cells under stress. Severe growth inhibition with higher astaxanthin synthesis activity may occur in the cells deprived of nitrogen, as in nitrogen-deplete cultures, resulting in overall reduced astaxanthin productivity. When nitrogen is in sufficient supply, like in the case of 8.8 and $17.6 \mathrm{mM}$ nitrate cultures, the cells can grow and reproduce while to a lesser extent synthesize astaxanthin under high light. Note that the optimal initial nitrogen concentration of $4.4 \mathrm{mM}$ nitrate is comparable to that applied to H. pluvialis culture maintained in an indoor aerated mini-reactor (Orosa et al. 2001), an indoor tube bioreactor (30 mm inner diameter, $70 \mathrm{~mL}$ culture volume) (Fábregas et al. 2001) and an indoor vertical bubble column $(70 \mathrm{~mm}$ inner diameter, 1.8 L culture volume) (García-Malea et al. 2005). Regardless the nitrogen availability, the maximum biomass productivity of all the cultures was obtained on day 2 or 3 of the red stage, whereas the maximum astaxanthin content 

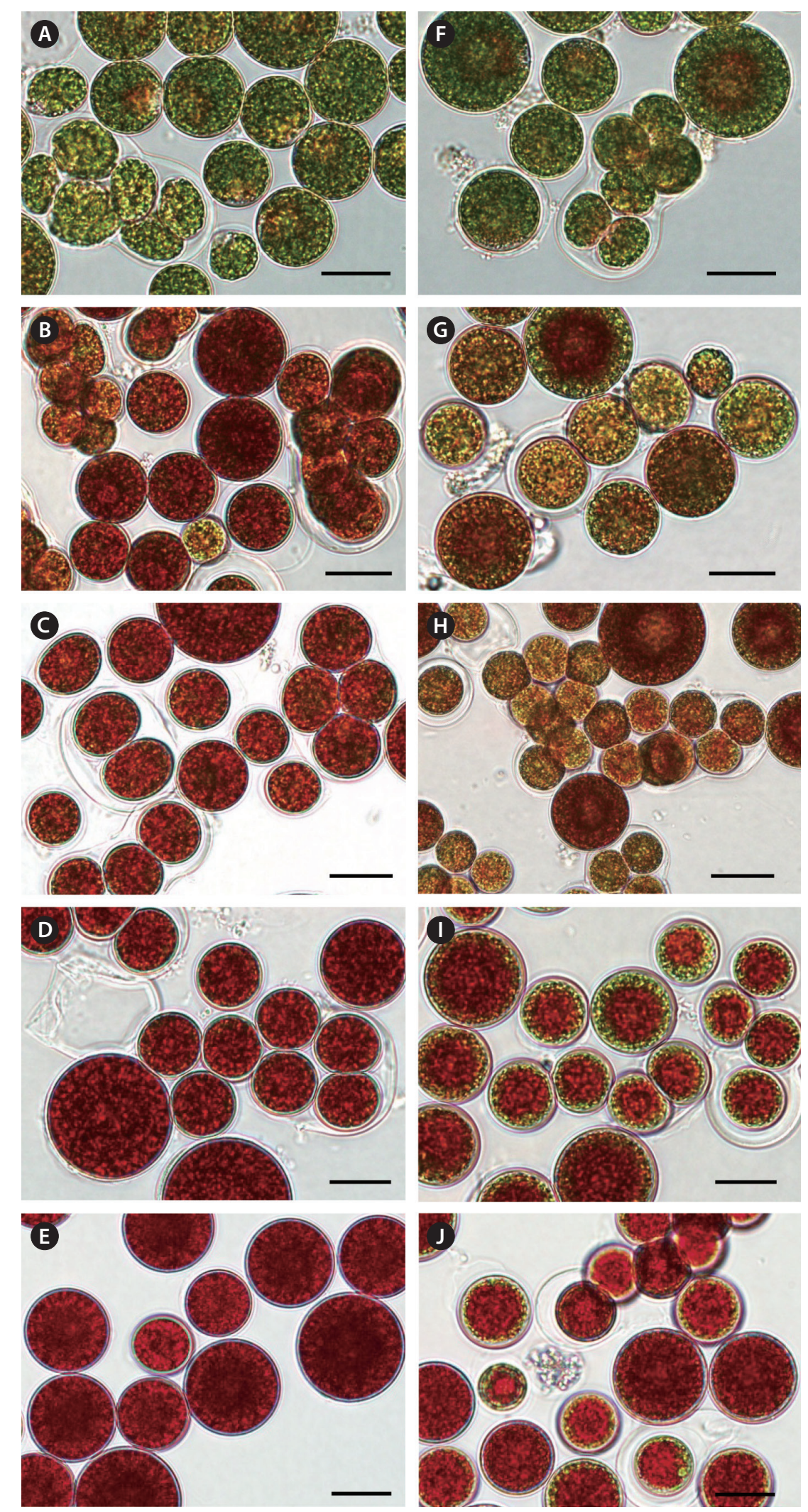

Fig. 4. Photomicrographs of Haematococcus pluvialis cells grown in outdoor glass columns containing 0 or $17.6 \mathrm{mM}$ nitrate medium. Cell images $A, B, C, D$, and $E$ were taken on days $1,3,4,7$, and 10 of the nitrogen-deplete culture, respectively. Cell images $F, G, H$, I, and J were taken on days $1,3,4,7$, and 10 of the $17.6 \mathrm{mM}$ nitrate culture, respectively. Scale bars represent: A-J, $20 \mu \mathrm{m}$. 

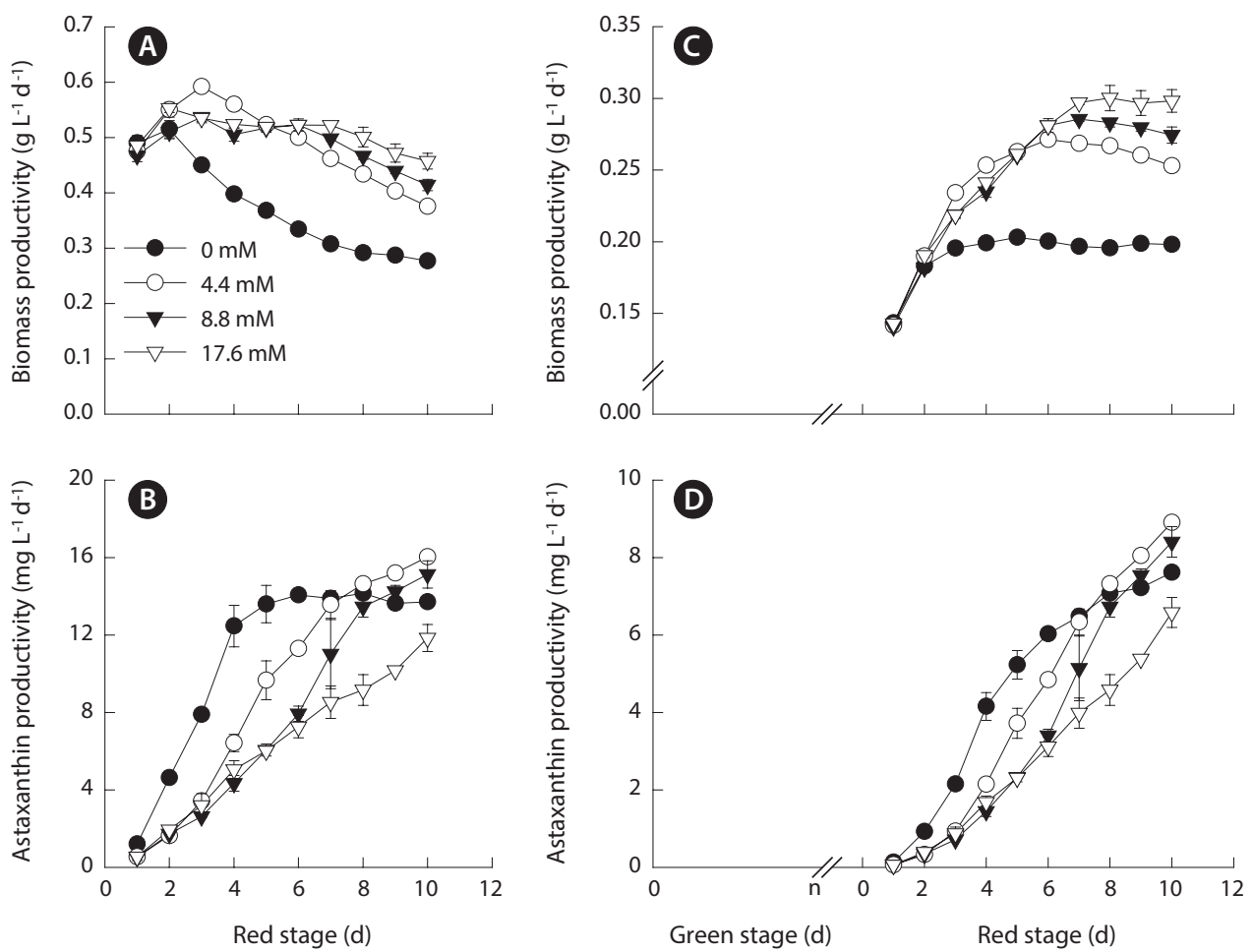

Fig. 5. Effects of nitrate concentrations on the biomass productivity and astaxanthin productivity of Haematococcus pluvialis under outdoor conditions. (A \& B) Results on the red stage. (C \& D) Results on green + red stages. The green stage duration was $n=8$ days. Data were means \pm standard deviations of six measurements from three individual columns (two measurements per column). The values in the legend represent different initial nitrogen concentration treatments. The initial biomass density was $0.8 \mathrm{~g} \mathrm{~L}^{-1}$ for this experiment.

occurred on day 10 or longer. At the red stage, the highest astaxanthin productivity of $16.0 \pm 0.15 \mathrm{mg} \mathrm{L}^{-1} \mathrm{~d}^{-1}$ was obtained in the $4.4 \mathrm{mM}$ nitrate cultures (Fig. 5B).

When the green stage was taken into account, the maximum volumetric biomass productivity of the nitrate-deplete cultures was $0.20 \pm 0.002 \mathrm{~g} \mathrm{~L}^{-1} \mathrm{~d}^{-1}$. When the initial nitrate concentrations were 4.4, 8.8, and $17.6 \mathrm{mM}$, the maximum biomass productivities increased to $0.27 \pm$ $0.004,0.29 \pm 0.002$, and $0.30 \pm 0.01 \mathrm{~g} \mathrm{~L}^{-1} \mathrm{~d}^{-1}$, respectively (Fig. 5C). The maximum astaxanthin productivity of 8.9 $\pm 0.08 \mathrm{mg} \mathrm{L}^{-1} \mathrm{~d}^{-1}$ was obtained in the $4.4 \mathrm{mM}$ nitrate cultures, and reduced astaxanthin productivities occurred in cultures with lower or higher nitrogen concentrations (Fig. 5D).

Developing an understanding of the combined effect of algal biomass density and nitrogen availability on growth and astaxanthin synthesis of $H$. pluvialis has far-reaching biotechnological implications. It has been widely considered that nitrogen depletion combined with high light stress is the most effective method for maximum astaxanthin induction (Borowitzka et al. 1991, Boussiba and Vonshak 1991, Fábregas et al. 2001, Torzillo et al. 2003,
García-Malea et al. 2006, Aflalo et al. 2007). In this study, we demonstrated that the IBD and initial nitrogen concentration that lead to highest growth are different from that resulting in the highest cellular astaxanthin content. In other words, a combination of any IBD and initial nitrogen concentration would not simultaneously result in both maximum growth of and greatest cellular astaxanthin content in H. pluvialis. Maximum astaxanthin productivity is, therefore, a tradeoff between the two parameters. To ensure maximum astaxanthin productivity is the primary goal of Haematococcus mass culture, especially when the Haematococcus biomass is used as a food or feed additive. However, if astaxanthin rather than whole cell biomass is the end product, then maximum cellular astaxanthin content may be more critical, as it affects the efficiency and thus cost of astaxanthin extraction and purification from Haematococcus biomass. In the latter case, an optimal IBD in a nitrogen deplete culture medium during the red stage would be the best conditions for achieving maximum cellular astaxanthin content, albeit at somewhat reduced biomass productivity. 


\section{CONCLUSIONS}

The maximum biomass production and maximum cellular astaxanthin content of $H$. pluvialis are mutually exclusive. The highest astaxanthin content of $3.8 \%$ of dry weight was obtained in nitrogen-deplete, and in IBD of 0.5-0.8 $\mathrm{g} \mathrm{L}^{-1}$ cultures, whereas the maximum volumetric biomass productivity of $0.30 \mathrm{~g} \mathrm{~L}^{-1}$ occurred in the same IBD but with nitrogen-replete cultures. When the two parameters were compromised (i.e., $0.8 \mathrm{~g} \mathrm{~L}^{-1} \mathrm{IBD}$ and $4.4 \mathrm{mM}$ nitrate), the maximum astaxanthin productivity of $18.71 \pm 2.69 \mathrm{mg} \mathrm{L}^{-1} \mathrm{~d}^{-1}$ was obtained at the red stage of Haematococcus culture. It therefore would be the end product, either astaxanthin or whole Haematococcus biomass, that determines a specific IBD and initial nitrogen concentration that should be applied to red stage cultivation to achieve maximum economic profitability.

\section{ACKNOWLEDGEMENTS}

The authors thank Dr. Danxiang Han for her scientific advice and critical reading of the manuscript.

\section{REFERENCES}

Aflalo, C., Meshulam, Y., Zarka, A. \& Boussiba, S. 2007. On the relative efficiency of two- $v s$. one-stage production of astaxanthin by the green alga Haematococcus pluvialis. Biotechnol. Bioeng. 98:300-305.

Borowitzka, M. A., Huisman, J. M. \& Osborn, A. 1991. Culture of the astaxanthin-producing green alga Haematococcus pluvialis. 1. Effects of nutrients on growth and cell type. J. Appl. Phycol. 3:295-304.

Boussiba, S. \& Vonshak, A. 1991. Astaxanthin accumulation in the green alga Haematococcus pluvialis. Plant Cell Physiol. 32:1077-1082.

Fábregas, J., Otero, A., Maseda, A. \& Domínguez, A. 2001. Two-stage cultures for the production of astaxanthin from Haematococcus pluvialis. J. Biotechnol. 89:65-71.

García-Malea, M. C., Acién, F. G., Fernández, J. M., Cerón, M. C. \& Molina, E. 2006. Continuous production of green cells of Haematococcus pluvialis: modeling of the irradiance effect. Enzyme Microb. Technol. 38:981-989.

García-Malea, M. C., Brindley, C., Del Río, E., Acién, F. G., Fernández, J. M. \& Molina, E. 2005. Modelling of growth and accumulation of carotenoids in Haematococcus pluvialis as a function of irradiance and nutrients supply. Biochem. Eng. J. 26:107-114.
García-Malea López, M. C., Del Río Sánchez, E., Casas López, J. L., Acién Fernández, F. G., Fernández Sevilla, J. M., Rivas, J., Guerrero, M. G. \& Grima, E. M. 2006. Comparative analysis of the outdoor culture of Haematococcus pluvialis in tubular and bubble column photobioreactors. J. Biotechnol. 123:329-342.

Guerin, M., Huntley, M. E. \& Olaizola, M. 2003. Haematococcus astaxanthin: applications for human health and nutrition. Trends Biotechnol. 21:210-216.

Han, D., Li, Y. \& Hu, Q. 2013. Biology and large-scale production of Haematococcus pluvialis. In Richmond, A. \& Hu, Q. (Eds.) Handbook of Microalgal Culture. 2nd ed. WileyBlackwell, Chichester, WS, pp. 388-405.

Han, D., Wang, J. F., Sommerfeld, M. \& Hu, Q. 2012. Susceptibility and protective mechanisms of motile and nonmotile cells of Haematococcus pluvialis (Chlorophyceae) to photooxidative stress. J. Phycol. 48:693-705.

Hu, Q. \& Richmond, A. 1996. Productivity and photosynthetic efficiency of Spirulina platensis as affected by light intensity, algal density and rate of mixing in a flat plate photobioreactor. J. Appl. Phycol. 8:139-145.

Hu, Q., Zarmi, Y. \& Richmond, A. 1998. Combined effects of light intensity, light-path and culture density on output rate of Spirulina platensis (Cyanobacteria). Eur. J. Phycol. 33:165-171.

Johnson, E. A. \& An, G. -H. 1991. Astaxanthin from microbial sources. Crit. Rev. Biotechnol. 11:297-326.

Karppi, J., Rissanen, T. H., Nyyssönen, K., Kaikkonen, J., Olsson, A. G., Voutilainen, S. \& Salonen, J. T. 2007. Effects of astaxanthin supplementation on lipid peroxidation. Int. J. Vitam. Nutr. Res. 77:3-11.

Kobayashi, M. 2000. In vivo antioxidant role of astaxanthin under oxidative stress in the green alga Haematococcus pluvialis. Appl. Microbiol. Biotechnol. 54:550-555.

Lichtenthaler, H. K. \&Wellburn, A. R. 1983. Determination of total carotenoids and chlorophyll $a$ and $b$ of leaf extracts in different solvents. Biochem. Soc. Trans. 603:591-592.

Lorenz, R. T. \& Cysewski, G. R. 2000. Commercial potential for Haematococcus microalgae as a natural source of astaxanthin. Trends Biotechnol. 18:160-167.

Miki, W. 1991. Biological functions and activities of animal carotenoids. Pure Appl. Chem. 63:141-146.

Nishino, H. 1998. Cancer prevention by carotenoids. Mutat. Res. 402:159-163.

Orosa, M., Franqueira, D., Cid, A. \& Abalde, J. 2001. Carotenoid accumulation in Haematococcus pluvialis in mixotrophic growth. Biotechnol. Lett. 23:373-378.

Stanier, R. Y., Kunisawa, R., Mandel, M. \& Cohen-Bazire, G. 1971. Purification and properties of unicellular bluegreen algae (order Chroococcales). Bacteriol. Rev. 
35:171-205.

Stirbet, A., \& Govindjee. 2011. On the relation between the Kautsky effect (chlorophyll $a$ fluorescence induction) and photosystem II: basic and applications of the OJIP fluorescence transient. J. Photochem. Photobiol. B 104:236-257.

Torzillo, G., Goksan, T., Faraloni, C., Kopecky, J. \& Masojídek, J. 2003. Interplay between photochemical activities and pigment composition in an outdoor culture of Haematococcus pluvialis during the shift from the green to red stage. J. Appl. Phycol. 15:127-136.

Wang, J., Han, D., Sommerfeld, M. R., Lu, C. \& Hu, Q. 2013. Effect of initial biomass density on growth and astaxanthin production of Haematococcus pluvialis in an outdoor photobioreactor. J. Appl. Phycol. 25:253-260.

Zhang, B. Y., Geng, Y. H., Li, Z. K., Hu, H. J. \& Li, Y. G. 2009. Production of astaxanthin from Haematococcus in open pond by two-stage growth one-step process. Aquaculture 295:275-281. 